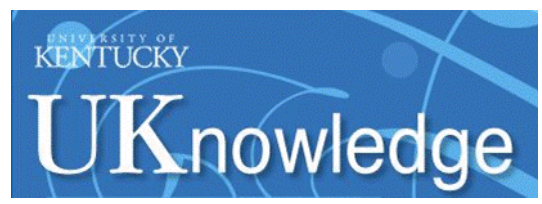

University of Kentucky

UKnowledge

\title{
Single Molecule-Level Study of Donor-Acceptor Interactions and Nanoscale Environment in Blends
}

Nicole Quist

Oregon State University

Rebecca Grollman

Oregon State University

Jeremy Rath

Oregon State University

Alex Robertson

Oregon State University

Michael Haley

University of Oregon

See next page for additional authors

Follow this and additional works at: https://uknowledge.uky.edu/chemistry_facpub

Part of the Chemistry Commons, Nanoscience and Nanotechnology Commons, and the Semiconductor and Optical Materials Commons

Right click to open a feedback form in a new tab to let us know how this document benefits you.

\section{Repository Citation}

Quist, Nicole; Grollman, Rebecca; Rath, Jeremy; Robertson, Alex; Haley, Michael; Anthony, John E.; and Ostroverkhova, Oksana, "Single Molecule-Level Study of Donor-Acceptor Interactions and Nanoscale Environment in Blends" (2017). Chemistry Faculty Publications. 116.

https://uknowledge.uky.edu/chemistry_facpub/116

This Conference Proceeding is brought to you for free and open access by the Chemistry at UKnowledge. It has been accepted for inclusion in Chemistry Faculty Publications by an authorized administrator of UKnowledge. For more information, please contact UKnowledge@lsv.uky.edu. 


\title{
Single Molecule-Level Study of Donor-Acceptor Interactions and Nanoscale Environment in Blends
}

\author{
Digital Object Identifier (DOI) \\ https://doi.org/10.1117/12.2251276
}

\section{Notes/Citation Information}

Published in Proceedings of SPIE, v. 10101, Organic Photonic Materials and Devices XIX, article 101010K, p. 1-8.

Copyright 2017 Society of Photo-Optical Instrumentation Engineers (SPIE). One print or electronic copy may be made for personal use only. Systematic reproduction and distribution, duplication of any material in this paper for a fee or for commercial purposes, or modification of the content of the paper are prohibited.

Nicole Quist , Rebecca Grollman , Jeremy Rath , Alex Robertson, Michael Haley, John Anthony, Oksana Ostroverkhova, "Single molecule-level study of donor-acceptor interactions and nanoscale environment in blends," Proc. SPIE 10101, Organic Photonic Materials and Devices XIX, 101010K (16 February 2017). DOI: https://doi.org/10.1117/12.2251276

The copyright holder has granted the permission for posting the article here.

\section{Authors}

Nicole Quist, Rebecca Grollman, Jeremy Rath, Alex Robertson, Michael Haley, John E. Anthony, and Oksana Ostroverkhova 


\section{Single molecule-level study of donor- acceptor interactions and nanoscale environment in blends}

Nicole Quist, Rebecca Grollman, Jeremy Rath, Alex Robertson, Michael Haley, et al.

Nicole Quist, Rebecca Grollman, Jeremy Rath, Alex Robertson, Michael Haley, John Anthony, Oksana Ostroverkhova, "Single molecule-level study of donor-acceptor interactions and nanoscale environment in blends," Proc. SPIE 10101, Organic Photonic Materials and Devices XIX, 101010K (16 February 2017); doi: 10.1117/12.2251276

SPIE. Event: SPIE OPTO, 2017, San Francisco, California, United States 


\title{
Single molecule-level study of donor-acceptor interactions and nanoscale environment in blends
}

\author{
Nicole Quist ${ }^{\mathrm{a}}$, Rebecca Grollman ${ }^{\mathrm{a}}$, Jeremy Rath ${ }^{\mathrm{a}}$, Alex Robertson ${ }^{\mathrm{a}}$, Michael Haley ${ }^{\mathrm{b}}$, John \\ Anthony $^{\mathrm{c}}$, and Oksana Ostroverkhova ${ }^{\mathrm{a}}$ \\ ${ }^{a}$ Oregon State University, Corvallis, Oregon, United States \\ ${ }^{\mathrm{b}}$ University of Oregon, Eugene, Oregon, United States \\ ${ }^{c}$ University of Kentucky, Lexington, Kentucky, United States
}

\begin{abstract}
Organic semiconductors have attracted considerable attention due to their applications in low-cost (opto)electronic devices. The most successful organic materials for applications that rely on charge carrier generation, such as solar cells, utilize blends of several types of molecules. In blends, the local environment strongly influences exciton and charge carrier dynamics. However, relationship between nanoscale features and photophysics is difficult to establish due to the lack of necessary spatial resolution. We use functionalized fluorinated pentacene (Pn) molecule as single molecule probes of intermolecular interactions and of the nanoscale environment in blends containing donor and acceptor molecules. Single Pn donor (D) molecules were imaged in PMMA in the presence of acceptor (A) molecules using wide-field fluorescence microscopy. Two sample configurations were realized: (i) a fixed concentration of Pn donor molecules, with increasing concentration of acceptor molecules (functionalized indenofluorene or PCBM) and (ii) a fixed concentration of acceptor molecules with an increased concentration of the Pn donor. The D-A energy transfer and changes in the donor emission due to those in the acceptor- modified polymer morphology were quantified. The increase in the acceptor concentration was accompanied by enhanced photobleaching and blinking of the Pn donor molecules. To better understand the underlying physics of these processes, we modeled photoexcited electron dynamics using Monte Carlo simulations. The simulated blinking dynamics were then compared to our experimental data, and the changes in the transition rates were related to the changes in the nanoscale environment. Our study provides insight into evolution of nanoscale environment during the formation of bulk heterojunctions.
\end{abstract}

Keywords: Single molecule fluorescence spectroscopy, organic semiconductors, Monte Carlo, fluorescence intermittency, photobleaching

\section{INTRODUCTION}

There is a significant interest in understanding photophysics of organic semiconductors due to their applications in low-cost (opto)electronic devices. ${ }^{1}$ Most of these devices rely on the ability of the material to generate and/or transport charge carriers, which often utilize donor-acceptor (D-A) blends and are strongly dependent not only on molecular structures, but also on the nanoscale morphology and local environment. ${ }^{2-4}$ The contribution of the latter to the photophysics is difficult to quantify due to a lack of nanoscale resolution afforded by typical characterization methods. Therefore, approaches that enable direct studies of the molecular photophysics depending on the nanoenvironment, in materials relevant for organic (opto)electronic devices, are necessary. In this contribution, we use single-molecule fluorescence spectroscopy to probe intermolecular interactions and effects of local environment on photophysics of functionalized acene derivatives which have been previously utilized in thin-film-transistors and in D-A blends. ${ }^{1,5}$ These belong to one of the most promising classes of organic semiconductors: solution-processable, high-mobility small-molecule organic materials. More specifically, we report on the photophysics of functionalized fluorinated pentacene (Pn-TCHS-F8) molecules used as the donor and their

Further author information: (Send correspondence to O.O.)

E-mail: oksana@science.oregonstate.edu, Telephone: 15417371679

Organic Photonic Materials and Devices XIX, edited by Christopher E. Tabor, François Kajzar,

Toshikuni Kaino, Yasuhiro Koike, Proc. of SPIE Vol. 10101, 101010K · @ 2017 SPIE

CCC code: $0277-786 \mathrm{X} / 17 / \$ 18 \cdot$ doi: $10.1117 / 12.2251276$

Proc. of SPIE Vol. 10101 101010K-1 
interaction with acceptor molecules (PCBM or functionalized indenofluorene derivatives (IF-TIPS)) and with acceptor-modified polymer environment, measured on the single-molecule level.

Single molecule fluorescence spectroscopy has been used as a tool to understand nanoscale interactions and environment in a variety of materials and systems. ${ }^{1,6}$ Because it requires high fluorescence quantum yield (QY) and high photostability from the single molecule reporters and extremely low fluorescence background from the surrounding environment, finding a relevant optoelectronic system for systematic studies represents a challenge. Here, we utilize highly fluorescent and stable Pn-TCHS-F8 molecules as single molecule fluorescent donors and non-fluorescent PCBM and IF-TIPS acceptors, all incorporated at varied concentrations into a non-fluorescent PMMA matrix.

Analysis of experimental single-molecule fluorescence trajectories has enabled better understanding of energy and charge transfer processes between the photoexcited molecule and other molecules in the surrounding environment. ${ }^{7-10}$ However, due to complexity of the nanoscale environment, quantitative assessment of structureproperty relationships is challenging. In order to gain physical insight into the dynamics of the nanoscale interactions, Monte Carlo simulations have been utilized to model the charge carrier transitions that determine the experimentally measured fluorescence time trajectories. ${ }^{11-14}$ In the present study, we use a modified Monte Carlo model to provide insight into photophysics of individual Pn-TCHS-F8 molecules, depending on their nanoscale environment. Understanding these interactions provides insight on the formation of bulk heterojunctions (BHJs), which are the basis for most successful organic solar cells and other organic optoelectronic devices. ${ }^{1}$

\section{EXPERIMENTAL}

\subsection{Materials}

In order to investigate changes in the nanoscale environment during the BHJ formation and its effect on molecular photophysics, we chose the fluorinated pentacene (Pn) with functionalized side groups of TCHS ((tricyclohexylsilyl)ethynyl), Pn-TCHS-F8, as our single molecule reporter and created two types of samples. The first type of sample was a fixed concentration of Pn-TCHS-F8, with acceptor molecules of either PCBM or IF-TIPS added at variety of concentrations, which allowed for variation in the average spacing between the Pn-TCHS-F8 donor and the acceptor molecules. The other type was a fixed concentration of the IF-TIPS acceptor molecules and a variety of concentrations of Pn-TCHS-F8 added. All molecules used here have been utilized in our previous studies of D-A BHJs in devices. ${ }^{5}$

PCBM and IF-TIPS were chosen as acceptor molecules because of their low LUMO energies $(3.7$ and $4.0 \mathrm{eV}$, respectively) as compared to that of Pn-TCHS-F8 $(3.54 \mathrm{eV})$ and their ultra-low fluorescence at the wavelength of our experiments $(633 \mathrm{~nm})$, which minimizes fluorescence background. Both donor and acceptor molecules were incorporated into a 20-nm thick host matrix of poly(methyl)methacrylate (PMMA). The base was a $1 \% \mathrm{wt}$ solution of PMMA in toluene and Pn-TCHS-F8 was added in multiples of $3.44 \times 10^{-10} \mathrm{M}$ ("1x" concentration) depending on what specific nanoscale environment in the film was desired (yielding an average donor-donor spacing ranging between 0.54 and $5.3 \mu \mathrm{m}$, which ensures that single molecules could be optically resolved). Acceptor molecules were added to these solutions in appropriate concentrations to achieve the desired average acceptor-acceptor spacing in the film, which was varied between 5 and $20 \mathrm{~nm}$. At $5 \mathrm{~nm}$ acceptor-acceptor spacing effects of efficient FRET between Pn-TCHS-F8 donor and acceptor molecules, which rapidly quenched the donor fluorescence if the D-A distance was below the FRET radius, were seen. To increase the probability of the donor placement outside of the FRET radius from the nearest acceptor, experiments were performed at higher donor concentrations (ranging between "6x" and "100x"). Experiments in samples with the acceptor-acceptor spacings of below $5 \mathrm{~nm}$ could not be carried out due to increased fluorescence background from acceptor molecules.

The samples were prepared on glass coverslip substrates which were soaked in a detergent and water solution overnight, then sonicated for 40 minutes in the same solution before they were rinsed thoroughly with deionized water and dried under $N_{2}$. The films were then deposited by spin-casting at $3000 \mathrm{rpm}$ for 50 seconds from 60 $\mu \mathrm{L}$ of solution. 


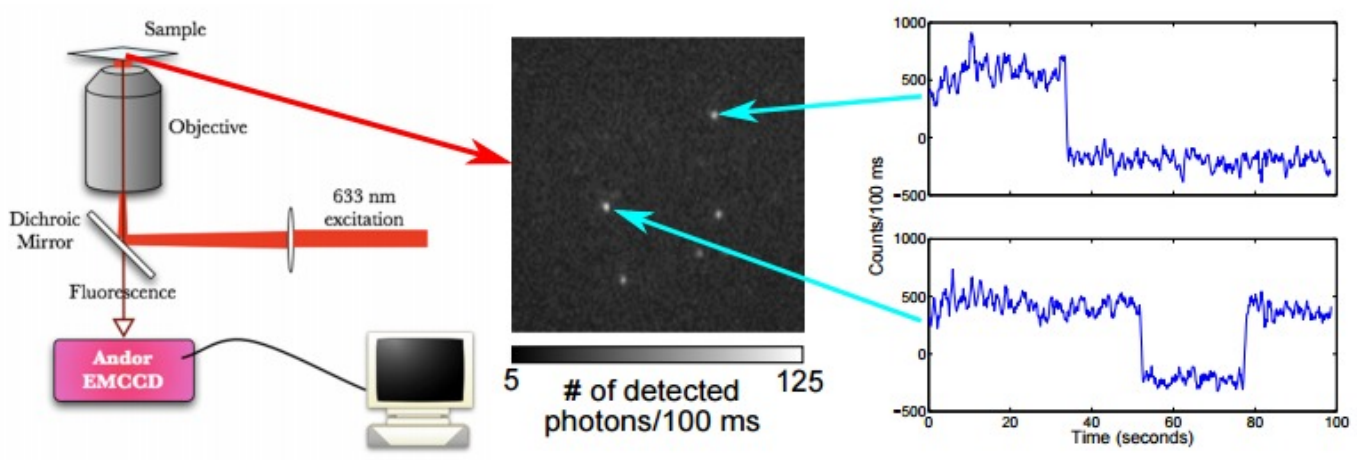

Figure 1. Left: Schematic of the experimental set up for single molecule florescence spectroscopy. Middle: Frame from the videos showing fluorescent single molecules. Right: Fluorescence time trajectories for a "non-blinking" molecule (top) and a "blinking" molecule (bottom).

\subsection{Single Molecule Spectroscopy}

Single molecule imaging was done using an Olympus IX-71 inverted microscope with a 100x UPlanSApo (NA 1.4) oil objective and an Andor iXon EMCCD (DU-897) detector. The sample was imaged using wide-field illumination from a $633 \mathrm{~nm}$ laser. Videos of 600 frames (with $100 \mathrm{~ms}$ integration time) were taken of each sample and then processed using custom MATLAB scripts (Fig 1). The MATLAB scripts located the fluorophores and created the fluorescence time trace for each fluorophore as detailed in our previous work. ${ }^{15}$ The time traces were analyzed, and fluorophores that exhibited only one "on"-"off" event ("non-blinkers") and multiple events ("blinkers") were separated.

The "on" and "off" times were determined using a custom MATLAB script. The last "off" or "on" time of each trace was discarded due to an uncertainty in their actual duration. The "on" times and "off" times for "blinkers", as well as "on" times for "non-blinkers" were compiled in three separate lists. The "on" and "off" times distributions were converted into complementary cumulative distribution functions (CCDFs) and then fit using maximum likelihood estimation (MLE) to various distribution functions including Weibull, lognormal and power-law (Fig. 2). ${ }^{16}$

\section{MONTE CARLO SIMULATION}

\subsection{The model}

In order to better understand the photophysical processes that are incorporated in the experimentally measured fluorescence time trajectories, we used a model to simulate the transitions between various states. Previous studies have used a three level system, with a ground state, excited state and dark state, to model the charge carrier kinetics as observed in single-molecule fluorescence experiments. ${ }^{11-14}$ In order to fully utilize our experimental findings and separate blinking events from photobleaching, we added a fourth, irreversible dark state which simulates photobleaching. Also guided by our experimental data, which show similarity of "on" times CCDFs for "blinkers" and "non-blinkers", is our choice of a common intermediate level (denoted as "dark states" in Fig.3) for "blinkers" and "non-blinkers", from which the molecule can either return to the ground state to be re-excited (for "blinker") or is permanently photobleached (for "non-blinker"). Because of long integration times used in our experiments, only long-lived ( $\geq 1$ second) dark states are considered.

Other photophysical data for Pn-TCHS-F8 are used to determine additional constraints for the model. For example, the fluorescence quantum yield (QY) for this derivative in PMMA is $0.82 .{ }^{15}$ This is the ratio of the number of photons emitted compared to the number of photons absorbed. In our model, only the transition between the excited state and the ground state radiates, and therefore the QY in a given time frame $(d t)$ can be defined as follows:

$$
Q Y=\frac{k_{21} * d t}{k_{2 t o t} * d t}=\frac{k_{21}}{k_{2 t o t}}
$$



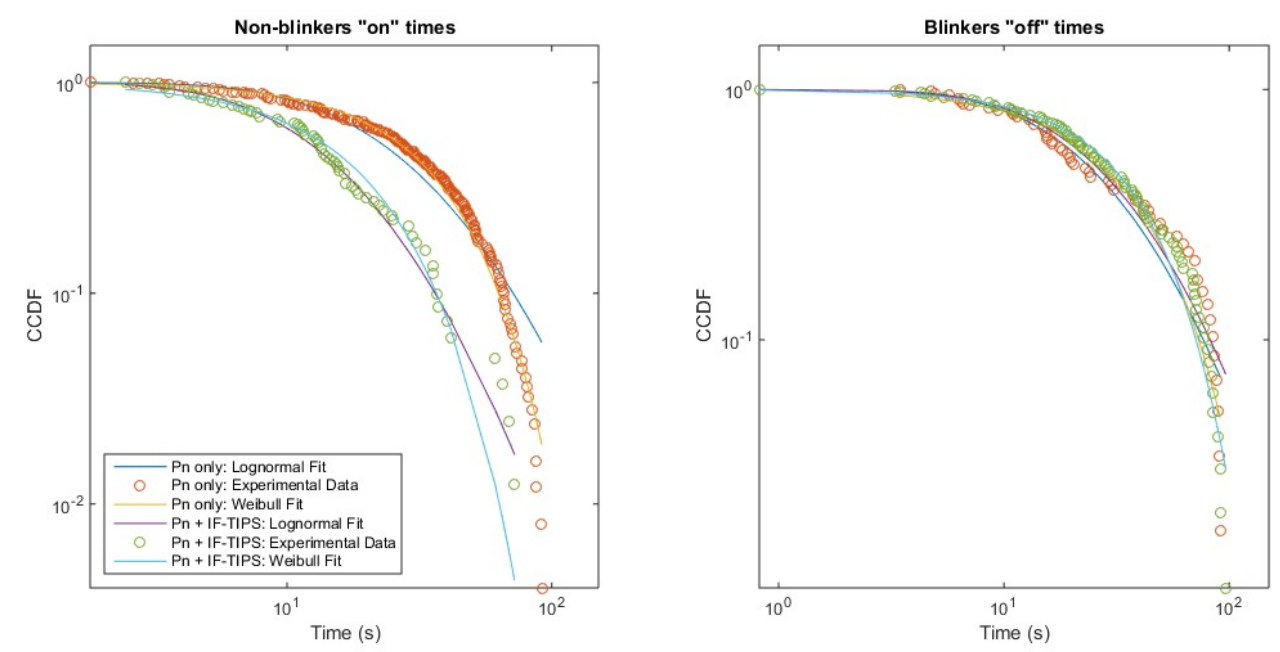

Figure 2. Complementary cumulative distribution functions of "on" times (left) and "off" times (right) from the experimental data for 78 "blinkers" and 251 "non-blinkers" in the case of Pn-TCHS-F8-only in PMMA and 122 "blinkers" and 81 "nonblinkers" for Pn-TCHS-F8 and 5 nm-spaced IF-TIPS acceptors in PMMA. MLE fits with Weibull and lognormal distribution functions are also included. The data illustrates dramatic effect of acceptor addition on the "on" times distribution exhibited by the Pn-TCHS-F8 single molecule reporters.

where $k_{21}$ is the transition rate between the excited state and the ground state and $k_{2 t o t}$ is the sum of the transition rates of all the transitions out of the excited state (or state 2). In our four level system, a QY of 0.82 indicates that $18 \%$ of the time, the system would either transition into the dark state or relax nonradiatively to the ground state. The average "on" times in our experimental data are on the order of 20-40 seconds at the excitation rate $k_{12}$ of $1.1 \times 10^{6}$. This makes the probability of transition to the long-lived dark state low $(<<1 \%)$, so that most of the $18 \%$ are due to nonradiative relaxation which was incorporated in the model using a rate $k_{21 n}$.

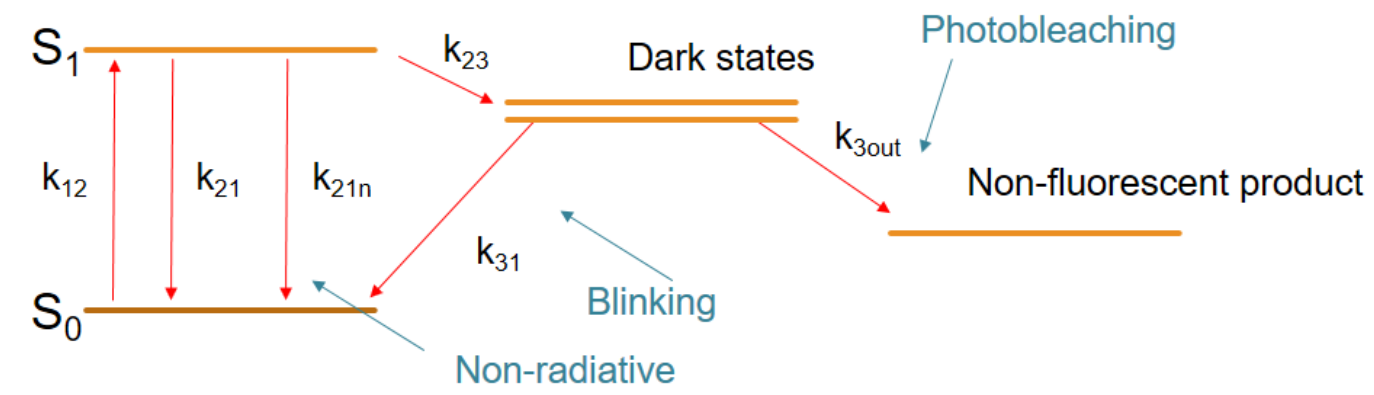

Figure 3. The four level system that includes the ground state, excited state, long-lived (reversible) dark state, and a photobleached (irreversible) dark state. Rates incorporated in the model are also included.

The transitions between energy levels in our four level system are simulated using a Monte Carlo method. In order for a transition to occur, the probability of transition must be determined first. The probability for a charge carrier in a given state to leave that state in a given time frame is given by

$$
P_{i j}=k_{i j} d t
$$


where $k_{i j}$ is the transition rate from the current state $i$ to the new state $j$. The calculated probability value is compared to a random number and if the probability value is greater than the random number, the transition occurs. This procedure becomes more complicated when there are multiple transition pathways out of a given state. In this case, the total probability of transition is calculated by summing the individual probabilities, and this value is compared with a random number to determine if a transition out of the state occurs. If the transition occurs, then the state it transitions to is determined by comparing ratios of transition rates (the QY, for example) with a random number.

To take into account variability in local nanoenvironment expected for polymer host matrices, ${ }^{11,12,14}$ we incorporated variable rates of transitions involving the dark state (i.e. $k_{23}$ and $k_{31}$ ) into our model following the approach of N. Wong et. al. ${ }^{12}$ In particular, a fixed transition rate was multiplied by $e^{\gamma * x}$, where $\gamma$ is a fixed integer coefficient and $x$ is a random number chosen from a Gaussian distribution with a mean of zero and a standard deviation of 0.5 .
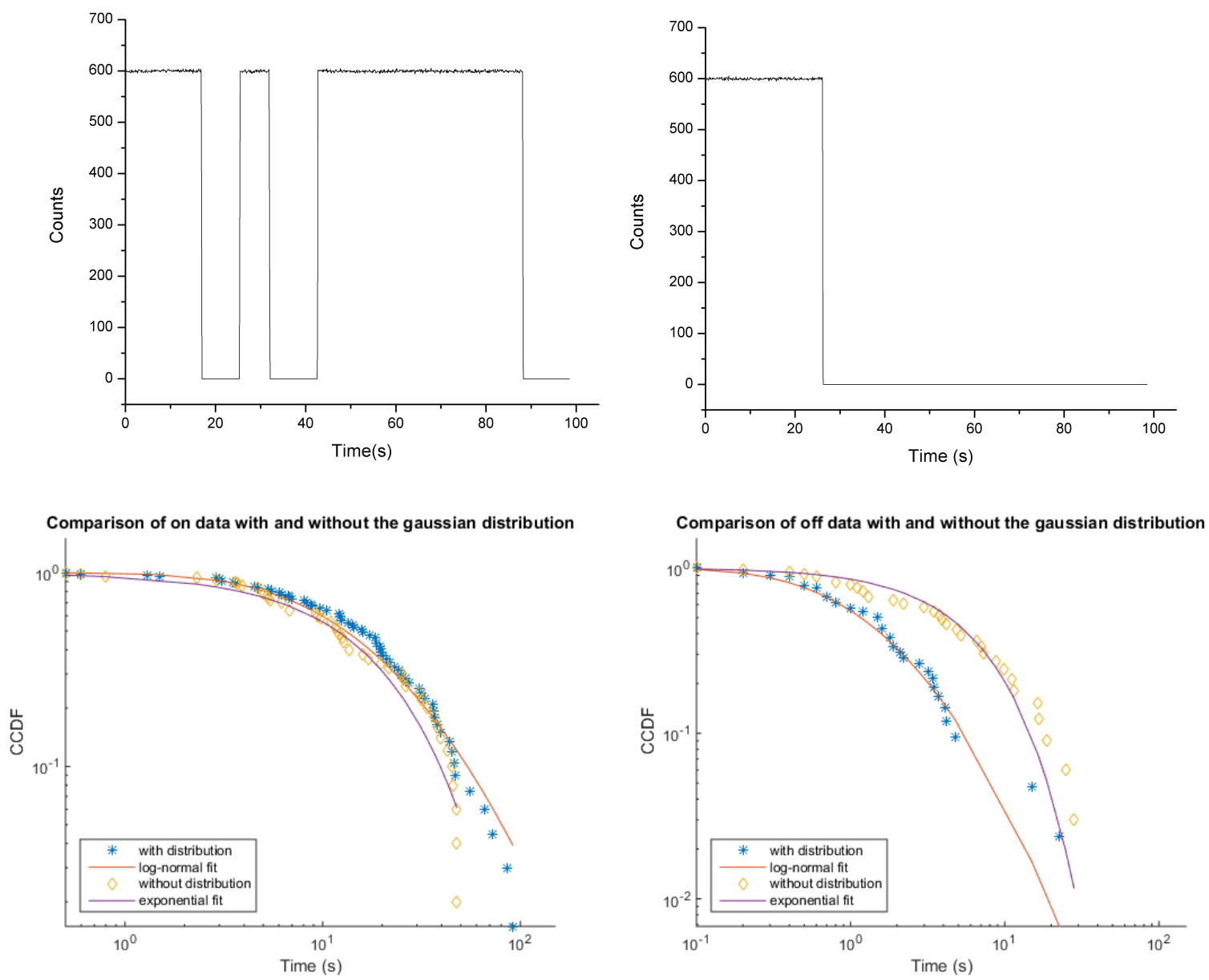

Figure 4. Top: Time traces from the Monte Carlo simulation of a "blinker" fluorophore (left) and a "non-blinker" fluorophore (right). Bottom: Complementary cumulative distribution functions (CCDFs) for "on" times (left) and "off" times (right) from 100 Monte Carlo time traces. This illustrates the change in shape of the CCDFs with the addition of the Gaussian distribution $(\gamma=5$ instead of 0$)$ for the $k_{23}$ and $k_{31}$ transition rates. The transition rates for these simulations were $k_{12}=1.1 \times 10^{6} s^{-1}, k_{21}=7.0 \times 10^{8} s^{-1}, k_{12 n}=1.53 \times 10^{8} s^{-1}, k_{23}=35 s^{-1}, k_{31}=0.05 s^{-1}$, and $k_{3 o u t}=0.1 s^{-1}$.

The simulation proceeds by preparing the system in the ground state, and it uses a $1 \mathrm{~ns}$ time step to step through the subsequent transitions. Every $0.1 \mathrm{~s}$, which is the EMCCD integration time used in our experiments, 
the photon counts are recorded, and a time trace similar to an experimentally observed fluorescence time trajectory is generated. The simulated time traces are processed using the same methods as our experimental data to produce CCDFs and MLE fits to various distribution functions (Fig. 4).
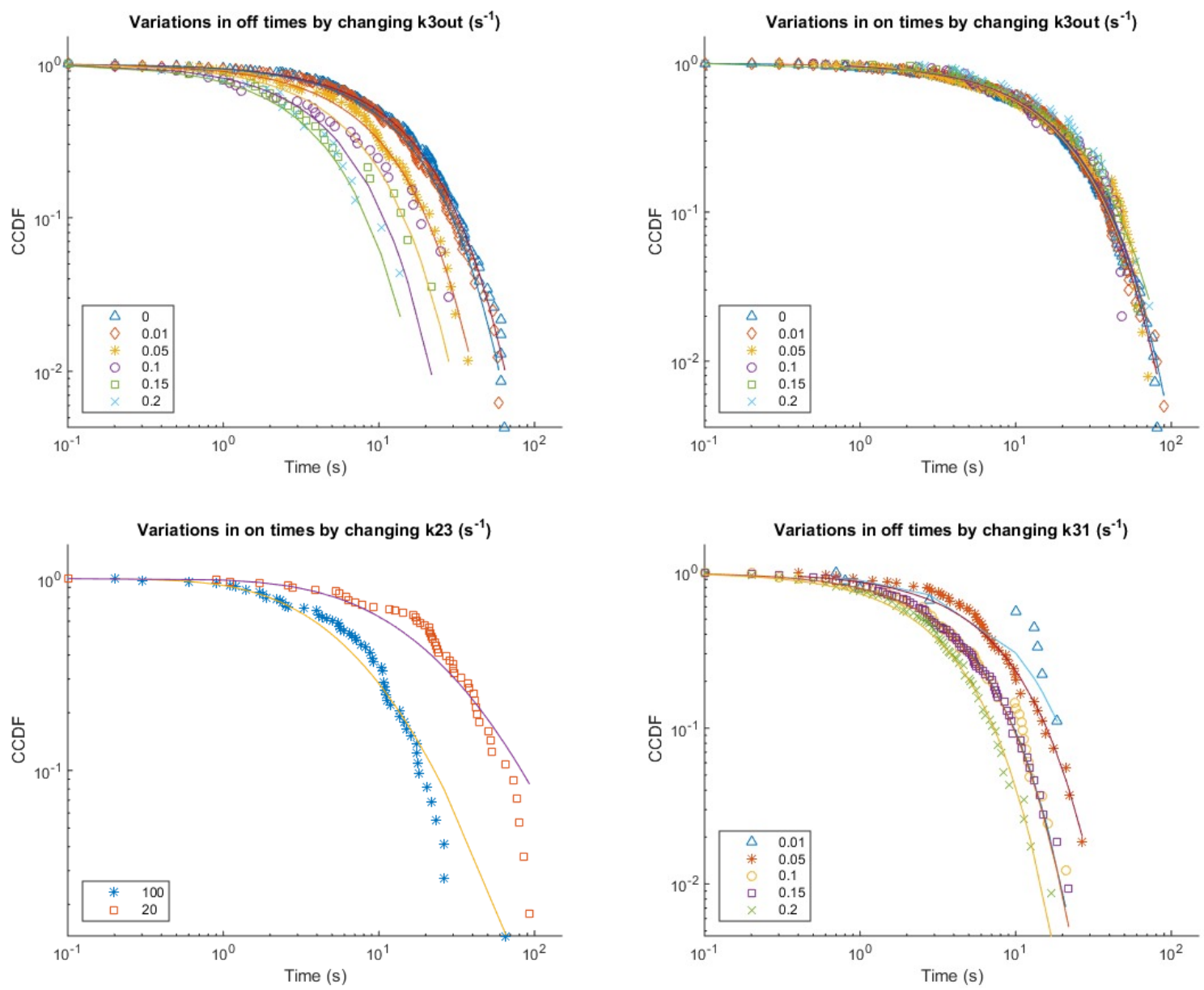

Figure 5. Top: Variations in the CCDFs of 100 Monte Carlo simulation time traces by varying the $k_{3 o u t}$ transition rate. As expected, the average "off" times (left) are significantly affected, in contrast to the "on" times (right). The fixed transition rates were $k_{12}=1.1 \times 10^{6} s^{-1}, k_{21}=7.0 \times 10^{8} s^{-1}, k_{12 n}=1.53 \times 10^{8} s^{-1}, k_{23}=35 s^{-1}, k_{31}=0.05 s^{-1}$, and $\gamma=0$. Bottom: Variations of the "on" times due to the change in $k_{23}$ (left), with $k_{12}=1.1 \times 10^{6} s^{-1}, k_{21}=7.0 \times 10^{8} s^{-1}$, $k_{12 n}=1.53 \times 10^{8} s^{-1}, k_{31}=0.05 s^{-1}, k_{3 o u t}=0.15 s^{-1}$, and $\gamma=3$. On the right, the variations in the "off" times due to the change in $k_{31}$, where $k_{12}=1.1 \times 10^{6} s^{-1}, k_{21}=7.0 \times 10^{8} s^{-1}, k_{12 n}=1.53 \times 10^{8} s^{-1}, k_{23}=35 s^{-1}, k_{3 o u t}=0.1 s^{-1}$, and $\gamma=0$. The $k_{3 o u t}$ and $k_{31}$ are fit to an exponential curves because they were run with fixed transition rates (no exponential multiplier). $k_{23}$ was fit to lognormal curves because they included the distibution.

\subsection{Testing}

Rigorous testing was carried out to determine the validity of the stimulation as well as the ways in which each parameter affects the time traces and the resulting CCDFs. Each parameter was varied while the other parameters remained fixed. For example, as the transition rate between the dark state and the irreversible (photobleached) state ( $k_{3 \text { out }}$ in Fig. 3 ) increased, the CCDF of the "off" times exhibited decays at earlier times, whereas the "on" times were not affected, as expected. Figure 5 also shows the dependence of the CCDF of the simulated data on the parameters $k_{23}$ and $k_{31}$, respectively, illustrating their pronounced effects on the "on" and "off" times distribution, respectively. These tests provide a valuable aid in understanding trends in our experimental data. 


\section{RESULTS AND DISCUSSION}

Figure 2 illustrates changes in the "on" and "off" times distributions for 203 (122 "blinkers" and 81 "nonblinkers") Pn-TCHS-F8 single-molecule reporters in PMMA with added 5 nm-spaced IF-TIPS acceptors, as compared to those for 329 (78 "blinkers" and 251 "non-blinkers") Pn-TCHS-F8 reporters alone in PMMA. The average "on" times for the samples containing IF-TIPS acceptors with an average acceptor-acceptor spacing of $5 \mathrm{~nm}$ are considerably shorter than those for the samples without acceptors, whereas the "off" times appear to be much less affected by the acceptor addition. Neither "on" nor "off" times follow the power-law distribution. Instead, depending on the sample, either Weibull or lognormal distributions provide a better fit to the data. For example, statistical analysis using the p-test indicates that in samples without acceptors, the "on" times distribution is best described by the Weibull function. Qualitatively, similar effects were observed upon addition of PCBM instead of IF-TIPS.

Our experimental results indicate that (i) the transition rates $k_{23}$ and $k_{31}$ are not constants, and their variability due to variations in the local nanoenvironment must be taken into account and (ii) the acceptor addition changes the environment in such a way that affects the rate $k_{23}$. The nature of transition to longlived dark states inferred from blinking statistics of molecules incorporated into polymer matrices has been discussed in the literature, ${ }^{10,12,17}$ with charge transfer to acceptor states in the polymer resulting in a change from a fluorescent parent molecule to a non-fluorescent radical ion as a possible mechanism. Given that in our experiments the Pn-TCHS-F8 single molecule reporters are positioned at least $3 \mathrm{~nm}$ away from the nearest IF-TIPS acceptor molecule, the observed effect of the IF-TIPS addition (Fig. 2) must be related to the IF-TIPSmodified polymer morphology and resulting distribution of the polymer acceptor states. Quantitative description of these changes is forthcoming.

\section{CONCLUSION}

In summary, using both computational modeling and single molecule fluorescence spectroscopy we investigated the nanoscale interactions of the Pn-TCHS-F8 with its environment modified by an addition of acceptor molecules. The model enables better understanding of relationships between the transition rates and the fit parameters obtained from the experimental CCDFs. In all samples studied, the blinking statistics observed could be described in the framework of models relying on Weibull or lognormal distribution functions relying on distributed (rather than constant) transition rates. We observed strong effect of an acceptor addition on the "on" time distribution governed by the rate $k_{23}$ for Pn-TCHS-F8 single molecules, whereas the "off" times (governed by $k_{31}$ and $k_{3 o u t}$ ) were considerably less affected. Quantitative description of such changes is currently

underway and will enable better insight into changes in nanoscale morphology and resulting photophysics during the formation of bulk heterojunctions.

\section{ACKNOWLEDGMENTS}

This work was supported by the National Science Foundation through grant DMR-1207309. N. Q. also acknowledges the SPIE Student Travel Grant Program, Oregon State University Graduate School and Oregon State University President's Commission on the Status of Women.

\section{REFERENCES}

[1] Ostroverkhova, O., "Organic optoelectronic materials: Mechanisms and applications," Chemical Reviews 116, 13279 - 13412 (2016).

[2] Paudel, K., Johnson, B., Neunzert, A., Thieme, M., Purushothaman, B., Payne, M. M., Anthony, J. E., and Ostroverkhova, O., "Small-molecule bulk heterojunctions: Distinguishing between effects of energy offsets and molecular packing on optoelectronic properties," Journal of Physical Chemistry C 117, 24752-24760 (2013). 
[3] Platt, A. D., Day, J., Subramanian, S., Anthony, J. E., and Ostroverkhova, O., "Optical, fluorescent, and (photo)conductive properties of high-performance functionalized pentacene and anthradithiophene derivatives," Journal of Physical Chemistry C 113, 14006-14014 (2009).

[4] Platt, A. D., Kendrick, M. J., Loth, M., Anthony, J. E., and Ostroverkhova, O., "Temperature dependence of exciton and charge carrier dynamics in organic thin films," Physical Review B 84, 235209 (2011).

[5] Kendrick, M. J., Neunzert, A., Payne, M. M., Purushothaman, B., Rose, B. D., Anthony, J. E., Haley, M. M., and Ostroverkhova, O., "Formation of the donor-acceptor charge-transfer exciton and its contribution to charge photogeneration and recombination in smallmolecule bulk heterojunctions," Journal of Physical Chemistry C 116, 18108-18116 (2012).

[6] Moerner, W. E., "A dozen years of single-molecule spectroscopy in physics, chemistry, and biophysics," Journal of Physical Chemistry B 106, 910-927 (2002).

[7] Ha, T. and Tinnefeld, P., "Photophysics of fluorescent probes for single-molecule biophysics and superresolution imaging," Annual Review of Physical Chemistry 63, 595-617 (2012).

[8] Riley, E. A., Bingham, C., Bott, E. D., Kahr, B., and Reid, P. J., "Two mechanisms for fluorescence intermittency of single violamine r molecules," Physical Chemistry Chemical Physics 13, 1879-1887 (2011).

[9] Hess, C. M., Riley, E. A., and Reid, P. J., "Dielectric dependence of single-molecule photoluminescence intermittency: Nile red in poly(vinylidene fluoride)," Journal of Physical Chemistry B 118, 8905-8913 (2014).

[10] Mitsui, M., Unno, A., and Azechi, S., "Understanding photoinduced charge transfer dynamics of single perylenediimide dyes in a polymer matrix by bin-time dependence of their fluorescence blinking statistics," Journal of Physical Chemistry C 120, 15070-15081 (2016).

[11] Wustholz, K. L., Bott, E. D., Isborn, C. M., Li, X., Kahr, B., and Reid, P. J., "Dispersive kinetics from single molecules oriented in single crystals of potassium acid phthalate," Journal of Physical Chemistry C 111, 9146-9156 (2007).

[12] Wong, N. Z., Ogata, A. F., and Wustholz, K. L., "Dispersive electron-transfer kinetics from single molecules on tio ${ }_{2}$ nanoparticle films," Journal of Physical Chemistry C 117, 21075-21085 (2013).

[13] Bott, E. D., Riley, E. A., Kahr, B., and Reid, P. J., "Proton-transfer mechanism for dispersed decay kinetics of single molecules isolated in potassium hydrogen phthalate," ACS Nano 3, 2403-2411 (2009).

[14] Maenosono, S., "Monte-carlo simulations of photoinduced fluorescence enhancement in semiconductor quantum dot arrays," Chemical Physics Letters 405, 182-186 (2005).

[15] Shepherd, W., Grollman, R., Robertson, A., Paudel, K., Hallani, R., Loth, M., Anthony, J., and Ostroverkhova, O., "Single-molecule imaging of organic semiconductors: Toward nanoscale insights into photophysics and molecular packing," Chemical Physics Letters 629, 29-35 (2015).

[16] Riley, E. A., Hess, C. M., Whitham, P. J., and Reid, P. J., "Beyond power laws: A new approach for analyzing single molecule photolminescence intermittency," The Journal of Chemical Physics 136, 184508 (2012).

[17] Haase, M., Hübner, C. G., Nolde, F., Müllen, K., and Basché, T., "Photoblinking and photobleaching of rylene diimide dyes," Physical Chemistry Chemical Physics 13, 1776-1785 (2011). 